\title{
Logistiksoftware IFIS UNO: Was haben wir gelernt?
}

\author{
Renato Lemm \\ Clemens Blattert \\ Hannes Aeberhard
}

Eidgenössische Forschungsanstalt für Wald, Schnee und Landschaft $(\mathrm{CH})^{*}$

Eidgenössische Forschungsanstalt für Wald, Schnee und Landschaft $(\mathrm{CH})$

Forst Aeberhard $\mathrm{GmbH}(\mathrm{CH})$

\begin{abstract}
Die Logistiksoftware IFIS UNO wurde von der Eidgenössischen Forschungsanstalt für Wald, Schnee und Landschaft (WSL) in Zusammenarbeit mit der Schweizer Forstwirtschaft und dem IT-Partner Ramco Systems entwickelt. Das System dient der Unterstützung und Optimierung der Geschäftsprozesse entlang der Forst-HolzKette. Nach beinahe zehn Jahren erfolgreichem Einsatz geht IFIS UNO Ende 2016 in den Ruhestand. IFIS UNO kann als Erfolgsgeschichte betrachtet werden. Durch Unterstützung von IFIS UNO konnten sich die Schweizer Holzvermarktungsorganisationen als zentrale und anerkannte Bündelungsnetzwerke etablieren. Gleichzeitig kurbelte es die Entwicklungen in diesem sehr spezifischen Softwarebereich an. Über die gewonnenen Erfahrungen aus der Entwicklung und dem laufenden Betrieb wird in diesem Beitrag berichtet.
\end{abstract}

doi: $10.3188 /$ szf.2016.0105

*Zürcherstrasse 111, CH-8903 Birmensdorf, E-Mail renato.lemm@wsl.ch
IFIS UNO ist ein Softwaresystem für die Holzlogistik. Es stellt die unternehmensübergreifende Kommunikation, Koordination und Kooperation unter den Akteuren in der Forst-Holz-Kette sicher und unterstützt diese bei der Vermarktung von Holz, einschliesslich dem zunehmend an Bedeutung gewinnenden Energieholz. IFIS UNO integriert dabei alle beteiligten Akteure wie Waldeigentümer, Förster, Forstunternehmer, Transporteure, Ver- marktungsorganisation sowie die Holzindustrie (Abbildung 1).

Mit IFIS UNO lassen sich u.a. die zeitund kostenintensive Bündelung des Holzangebotes optimieren und somit die Holznutzung im kleinstrukturierten Waldbesitz fördern. Weiter stärkt es die Position der Waldeigentümer am Holzmarkt, indem eine bessere Kundenorientierung ermöglicht wird, was wiederum zu einer effizienteren Allokation der Holzressourcen führt.

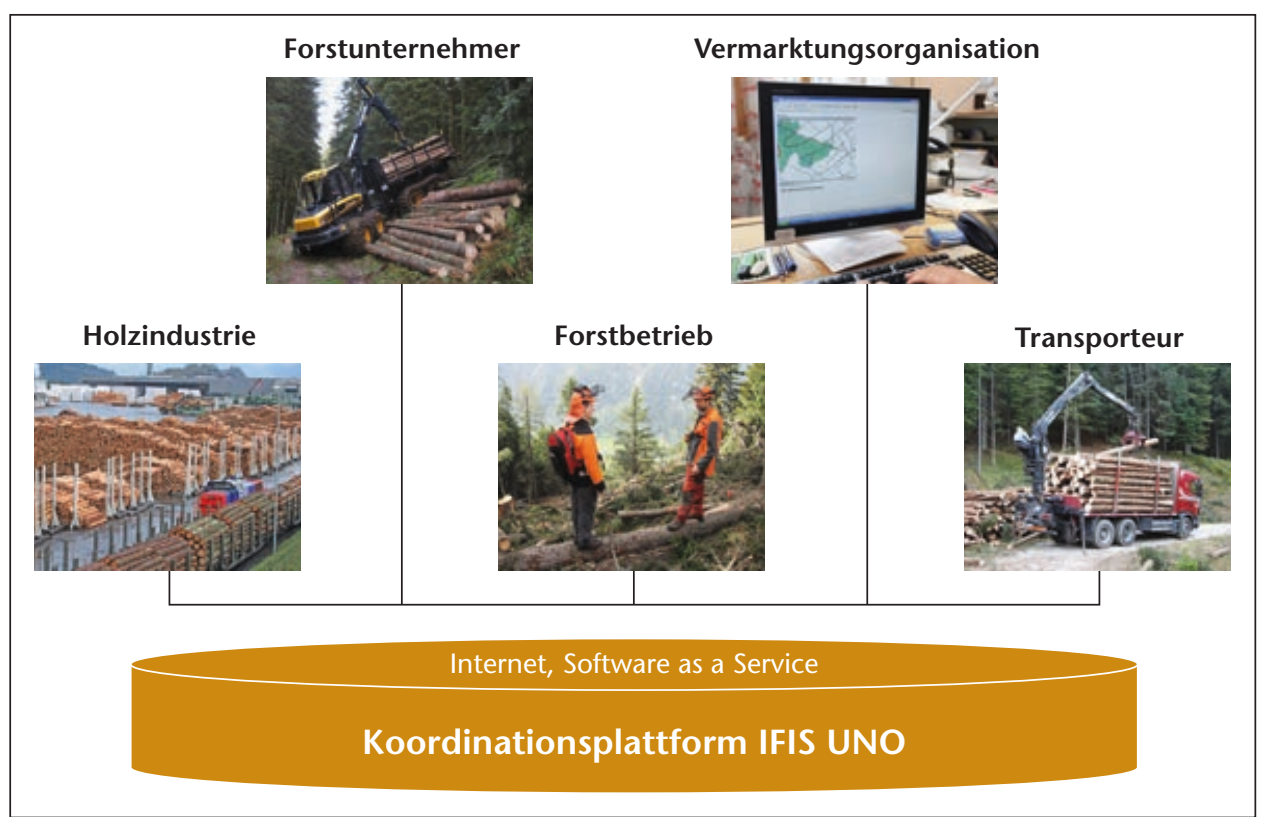

Abb 1 Die Plattform IFIS UNO ist ein Softwaresystem für die Holzlogistik. Sie wird über das Internet (Software as a Service, SaaS) angeboten (Lemm \& Thees 2009). Fotos: Ulrich Wasem (Holzindustrie), Verein IFIS (Vermarktungsorganisation), Fritz Frutig (restliche Bilder)
Das System wurde primär für Vermarktungsorganisationen entwickelt und ging im Juni 2007 bei der Aareholz AG, der ZürichHolz AG und der Holzvermarktungszentrale Nordwestschweiz HZN (heute Raurica Holzvermarktung AG) in den produktiven Betrieb. Wenig später schlossen sich weitere Nutzer, z.B. die Reziaholz $\mathrm{GmbH}$, an. Zeitweise wurden mehr als $500000 \mathrm{~m}^{3}$ Holz über IFIS UNO abgewickelt, was rund $10 \%$ des eingeschlagenen Holzes der Schweiz entspricht.

IFIS UNO basiert auf modernen Internettechnologien (Software as a service, SaaS) ${ }^{1}$ und ermöglicht dadurch eine einfache Bedienung und die schnelle Anpassung an neue Situationen. Die Lösung wurde massgeblich von der forstlichen Praxis mitgestaltet. Für die Entwicklung, den Vertrieb und den Unterhalt wurde als Geschäftsmodell die Vereinslösung gewählt und der Verein IFIS (Integrierte forstliche Informationssysteme) gegründet.

Eine Umfrage bei den Nutzern im Jahr 2008/2009 hatte gezeigt, dass die Funktionalitäten des Systems unterschiedlich

\footnotetext{
1 SaaS bezeichnet einen Teilbereich des Cloud-Computings. Vorteile sind: Es ist keine lokale Installation notwendig. Der Anwender muss sich nicht um Updates kümmern. Die Software lässt sich unabhängig vom Betriebssystem auf nahezu jedem Rechner und auf mobilen Geräten wie Notebooks, Tablets und Smartphones anwenden.
} 


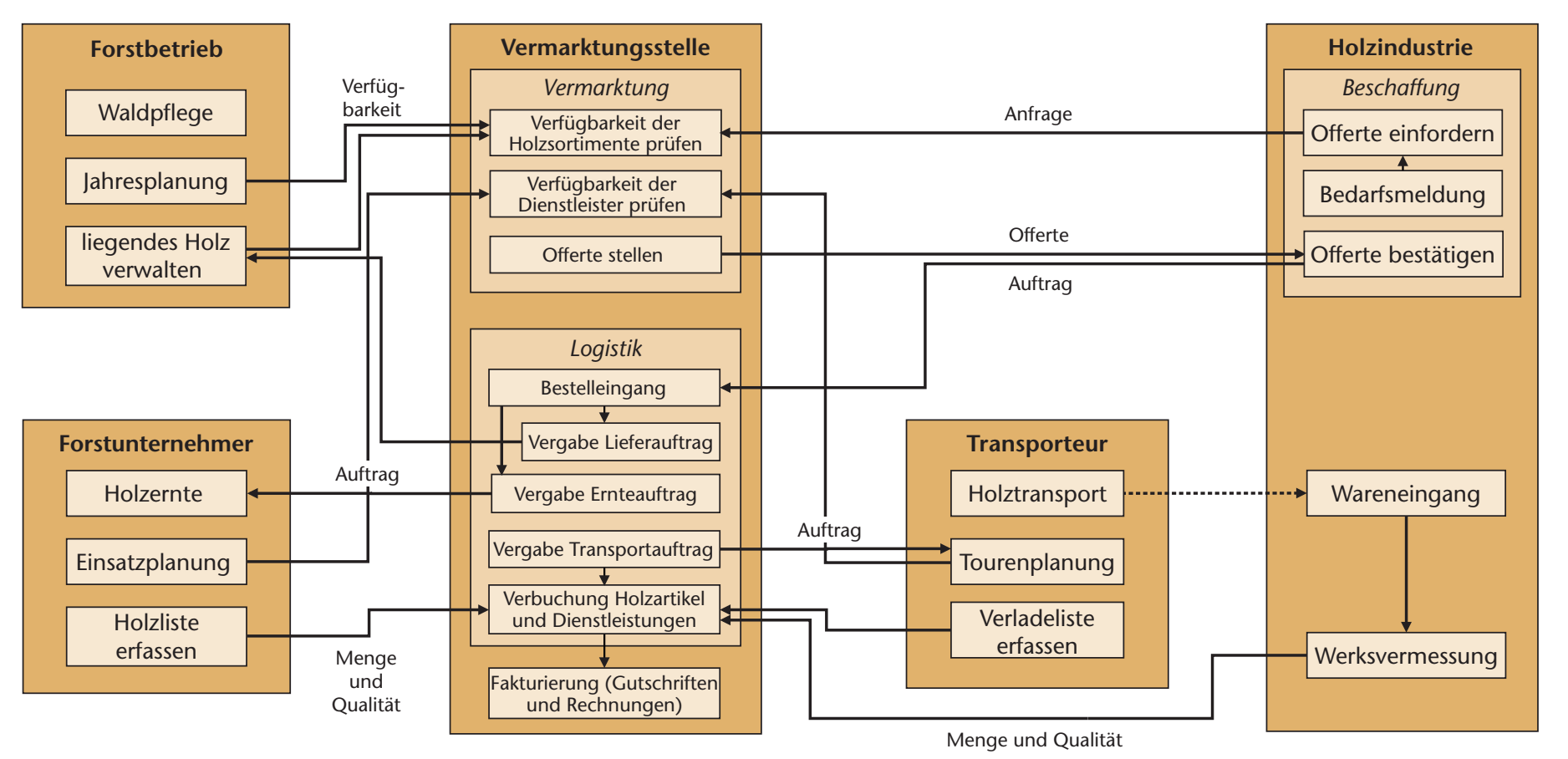

Abb 2 Zusammenspiel der Akteure, Rollen und Geschäftsabläufe der Holzvermarktung in IFIS UNO (Quade 2007).

genutzt werden. Aber alle Nutzer waren mit IFIS UNO zufrieden bis sehr zufrieden, da mit dessen Einsatz eine markante Effizienzsteigerung erzielt werden konnte.

\section{Anstoss für die Entwicklung}

Vor dem Hintergrund, die wirtschaftliche Situation der Waldbewirtschaftung in der Schweiz durch eine organisatorische Neugestaltung und den Einsatz moderner Informationstechnologien zu verbessern, hat die WSL im Jahr 2003 das Forschungsprogramm «Management zukunftsfähige Waldnutzung» gestartet. In dessen Rahmen wurde auch eine umfangreiche Evaluation durchgeführt mit dem Ziel, ein geeignetes System für die Holzvermarktung zu ermitteln. Dazu wurden die Geschäftsprozesse rund um den Verkauf und Vertrieb von Holz, von der Offertanfrage des Abnehmers über die waldseitige Verfügbarkeitsprüfung bis hin zum Transport und zur Abrechnung, gemeinsam mit den beteiligten Akteuren analysiert und optimiert. Dadurch wurde ersichtlich, wie und wo die Prozesse verknüpft sind (Abbildung 2). Die dokumentierten Prozesse dienten als Basis für die gezielte Evaluation bestehender Informationssysteme (Schnetzler et al 2007).

Die Evaluation zeigte, dass in der Schweiz und in den angrenzenden Nachbarländern kein System existierte, welches die vorgefundenen Geschäftsprozesse und die gewünschten funktionalen Anforderungen halbwegs befriedigen konnte (Lemm et al
2006). So sollte die Lösung über das Internet genutzt werden können und allen in der Forst-Holz-Kette beteiligten Akteuren nützlich sein. Insbesondere sollten durch Integration der zentralen Geschäftsprozesse entlang der Wertschöpfungskette Medienbrüche vermieden werden. Die WSL hat sich daher zusammen mit den zukünftigen Anwendern und einem IT-Partner dazu entschlossen, eine eigene Anwendung für die Vermarktung von Holz zu entwickeln.

\section{IFIS UNO und seine Zukunft}

Derzeit wird IFIS UNO noch von drei Vermarktungsorganisationen eingesetzt. Ende 2016 werden sie ihre Nutzung von IFIS UNO einstellen und auf ein anderes System wechseln. Gründe hierfür sind, dass der Softwareentwickler Ramco Systems seine Programmierung auf Indien zurückgezogen hatte, was die Kommunikation und die Fehlerbehebung erschwerte, und dass bei der Raurica Holzvermarktung AG verschiedene neue Geschäftsprozesse dazugekommen waren, die IFIS UNO nicht abbilden konnte und eine Nachprogrammierung zu teuer gewesen wäre. Die Raurica Holzvermarktung AG entschloss sich daher dazu, noch vor Ablauf der Vertragsfrist auf ein anderes Produkt umzusteigen. Für die ZürichHolz AG und Reziaholz GmbH war es in der Folge nicht mehr möglich, IFIS UNO finanziell zu tragen. Den 10-jährigen, reibungslosen Einsatz von IFIS UNO betrachten wir im Nachhinein als Erfolg, zumal gezeigt werden konnte, dass durch die Verwendung dieser Logistiksoftware die Geschäftsprozesse entlang der ForstHolz-Kette wesentlich verbessert werden konnten. Dies hat unter anderem den genannten Vermarktungsorganisationen eine zusätzliche Professionalität verliehen, wodurch sie am Holzmarkt an Bedeutung gewinnen konnten und sich als zentrale und anerkannte Bündelungsnetzwerke, auch über die Landesgrenzen hinweg, etablieren konnten. Weiter diente IFIS UNO anderen Anbietern ähnlicher Lösungen als positives Referenzobjekt und hat somit Innovationen in diesem Bereich massgeblich angekurbelt. Der Softwarehersteller Ramco erhielt für IFIS UNO auch einen Innovationspreis. Dabei wurde er für diese innovative ITLösung mit hohem Nutzwert aus über 5000 eingereichten Bewerbungen mit dem Prädikat BEST OF 2014 ausgezeichnet.

\section{Was haben wir während der Entwick-} lung und des Betriebs gelernt? Im Rahmen der Entwicklung und des Betriebs von IFIS UNO haben wir zahlreiche Erfahrungen gesammelt, die anderen bei ähnlichen Projekten hilfreich sein können. Die wesentlichsten stellen wir nachfolgend dar (vgl. auch Abbildung 3).

Vor- und Nachteile einer Individualsoftware sorgfältig prüfen

Eine Individualsoftware wird sinnvollerweise dann entwickelt, wenn sie Wettbe- 


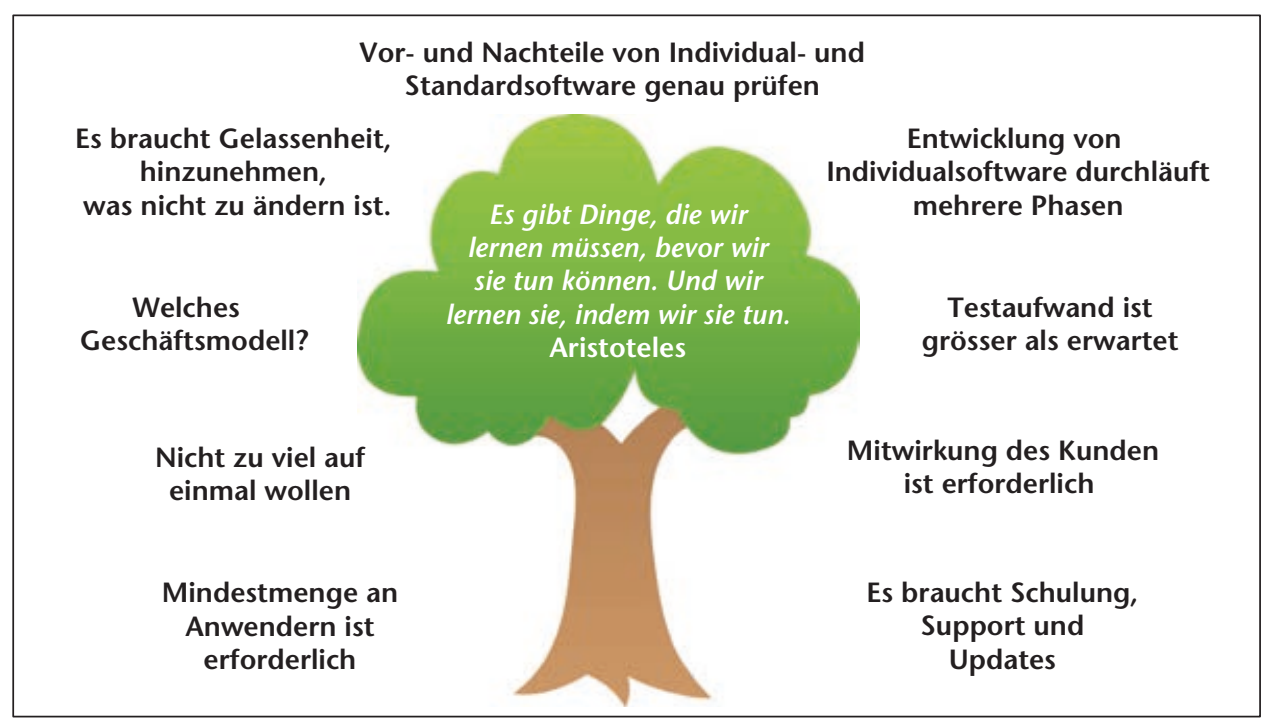

Abb 3 Was wir aus der Entwicklung und dem Betrieb von IFIS UNO gelernt haben.

\section{Vorteile}

Exakte Anpassung an die spezifische

Unternehmenspraxis

Keine Abhängigkeit vom Softwarehersteller

Keine laufenden Lizenz- und/oder

Wartungskosten

Keine vorgeschriebene Hard- und Software als Infrastruktur (gilt nur für SaaS-Lösung)

Weiterentwicklung frei in Zeit und Inhalt Know-how bezüglich Entwicklung, Support, Schulung wird aufgebaut

\section{Nachteile}

Möglicherweise hohe Entwicklungskosten

Bei jeder nachträglichen Änderung entstehen erneut Kosten und Testaufwände

Die Software ist erst nach einer Entwicklungsdauer einsatzfähig

Abhängigkeit vom Hersteller/Anbieter

Schulung der Anwender in Eigenregie

Entwicklung, Wartung und Betreuung der Software binden personelle Ressourcen und haben Kosten zur Folge

Weiterentwicklung kann beim Austritt von Mitarbeitern gefährdet sein

Software muss umfassend dokumentiert werden Weniger breite Anwenderbasis und weniger grosses Schulungsangebot (Wissensaustausch)

Tab 1 Vor- und Nachteile einer Individualsoftware gegenüber einer Standardlösung (Badertscher et al 2006, verändert).

werbsvorteile bringt oder die benötigte Software noch nicht existiert. Wie die Tabelle 1 zeigt, hat eine Individualsoftware gegenüber einer Lösung «von der Stange» verschiedene Vorteile, aber auch Nachteile. Daher ist die Frage, ob eine Individualsoftware entwickelt oder eine Standardsoftware eingeführt werden soll, genau zu prüfen.

Sich im Klaren sein, dass individuell entwickelte Software mehrere Phasen durchläuft Zu Beginn sind die Erwartungen und die Euphorie aller Beteiligten um die neue Lösung meistens gross. Doch vielfach macht sich bald eine gewisse Ernüchterung breit, da individuelle Wünsche oftmals nicht berücksichtigt werden können. Während der ersten Tests oder während
Zeitpunkt der Inbetriebnahme funktionieren? Was kann oder muss im Notfall warten? Ist diese Phase einmal durchgestanden, werden die Vorzüge der neuen Lösung langsam sichtbar. Zufriedenheit mit dem neuen System macht sich breit, und nach einiger Zeit wissen die Anwender, was sie zu tun haben, um ein gewünschtes Ergebnis zu erzielen.

\section{Der Testaufwand ist jeweils grösser als erwartet}

Nicht zu unterschätzen ist der Testaufwand. Der Testaufwand macht zwischen 20 und 70\% des Gesamtaufwandes aus. Ein vollständiges Testen auf verborgene Fehler im System ist allerdings nicht möglich, weshalb es mit Sicherheit nicht bei der ersten Systemversion bleiben wird. Es gilt daher, eine Testumgebung aufzubauen, die alle wesentlichen Funktionen der Anwendung beinhaltet. Diese Testumgebung kann später bei jedem grösseren Update verwendet werden, um zu prüfen, ob die wichtigsten Abläufe störungsfrei arbeiten.

\section{Mitwirkung des Anwenders erforderlich} Neben den eigentlichen Kosten für das Endprodukt muss der zukünftige Anwender gegenüber dem Entwickler einen zusätzlichen Input leisten, zum Beispiel muss er Informationen über sein bisheriges System bereitstellen und seine Geschäftsprozesse transparent darstellen. Zudem muss er seine Wünsche hinsichtlich der Funktionalitäten des Systems präzise formulieren. Unterschätzt ein Anwender diese Mitwirkungspflichten oder nimmt er diese nicht richtig wahr, sind Verzögerungen oder im schlimmsten Fall gar das Scheitern des Vorhabens die Folge. Die Mitwirkung muss daher präzise geregelt werden. Damit wird der tatsächliche Aufwand für beide Seiten transparent.

\section{Es braucht Schulung}

Die Schulung der nicht in die Entwicklung involvierten Mitarbeiter erfolgt sinnvollerweise dann, wenn das System getestet ist und stabil läuft. Schulungen an halbfertigen Lösungen, welche Mitarbeiter mehr verwirren, als sie auf die neue Umgebung vorzubereiten, müssen vermieden werden.

Weiter sollte sichergestellt werden, dass die Mitarbeiter auf das neue System vorbereitet werden. Dabei sollte unter Umständen auch erläutert werden, weshalb die neue Software eventuell noch nicht dort ist, wo 
sie sein sollte. Wichtig ist es, die Mitarbeiter einzubeziehen. Das Einführen von Tagungen zum Erfahrungsaustausch, wo die Nutzer über ihre Probleme diskutieren und gemeinsam nach Lösungen suchen, hat sich dabei als Erfolg herausgestellt.

\section{Support}

Mit der Überführung in den produktiven Betrieb gilt das System für den Entwickler in der Regel als abgeschlossen. Die meisten Systementwickler bieten für die weitere Arbeit entsprechende Supportverträge an. Für den Systembenutzer sind aber alle im Echtbetrieb noch auftretenden Fragen Bestandteil des Projektes. Dieser Übergang von der Projektvereinbarung zum Supportvertrag ist deshalb explizit zu regeln.

\section{Updates}

IT-Systeme müssen sich in einem realen, stetig verändernden Umfeld bewähren. Daher ist klar zu regeln, wie und zu welchen Konditionen der Softwarepartner notwendig gewordene Updates (z.B. als Folge neuer Abrechnungsformen oder Zuschläge) anbietet.

\section{Nicht zu viel auf einmal wollen}

Bei der Planung und Konzeption von IFIS UNO hatte man ein System entwerfen wollen, auf welches alle an der Forst- und Holzkette beteiligten Akteure zugreifen können. Im Nachhinein hat sich gezeigt, dass diese Vorstellung zu optimistisch war. Der Betreuungsaufwand, um alle Akteure zu bedienen, wäre viel zu gross gewesen. Es lohnt sich nicht, den grossen Wurf zu wollen. Sinnvoller ist es, in kleinen Schritten vorwärts zu gehen.

\section{Eine Mindestmenge an Anwendern ist erforderlich}

Die Entwicklung einer Software für die Holzlogistik muss durch die Anwender vorfinanziert werden. Dies ist nicht einfach, da eine auf die Schweizer Forstwirtschaft zugeschnittene IT-Lösung nie einen grossen Nutzerkreis umfassen wird und damit die Kosten für den einzelnen Akteur immer relativ hoch sein werden. Jede weitere Organisation, die hinzukommt, hilft jedoch, die Kosten pro Teilnehmer zu reduzieren. Leider ist die Expansion nicht so verlaufen, wie sich das der Verein IFIS vorgestellt hat. Eine Expansion in der Schweiz oder gar ins Ausland hätte finanzielle Mit- tel für weitere Innovationen einbringen können. Zu wachsen wurde zwar versucht, aber mit zu wenig Entschlossenheit.

\section{Welches Geschäftsmodell?}

Das Geschäftsmodell auf Vereinsbasis, wie es für IFIS UNO gewählt wurde, ist grundsätzlich Erfolg versprechend (Stephan et al 2009). Es braucht jedoch eine engagierte Person im Verein als Sprachrohr und als Vermittler zwischen den Systembenutzern und Systementwicklern für alle Belange der Nutzung, des Unterhalts und der Weiterentwicklung der Anwendung. Als Nachteil hat sich das teilweise vorhandene unprofessionelle» Image von Vereinen ausgewirkt. Es ist gut zu überlegen, ob nicht ein anderes Geschäftsmodell, zum Beispiel eine Gesellschaft mit beschränkter Haftung $(\mathrm{GmbH})$ oder eine Aktiengesellschaft (AG), zielführender wäre.

\section{Es braucht Gelassenheit, hinzunehmen, was nicht zu ändern ist}

Es ist äusserst frustrierend, wenn ein ITProjekt nicht wie geplant vorwärtskommt oder nicht fehlerfrei läuft. Die Ursache einer Störung muss aber nicht zwingend im neuen IT-System selbst liegen. Störungen und deren Ursachen sind daher selbstkritisch zu analysieren, und es sollte nach konstruktiven Lösungen gesucht werden. So muss geklärt werden, welche Punkte der weiteren Bearbeitung bedürfen, es sind Prioritäten festzulegen, und offene Punkte müssen mit dem Lieferanten besprochen werden. Lösungen und Termine sind, wo immer möglich, verbindlich zu vereinbaren. Der Abbruch des Projekts sollte immer vermieden werden. Im Zweifelsfalle ist es besser, den Dialog zu suchen und einen für beide Seiten vernünftigen Kompromiss zu finden.

\section{Literatur}

BADERTSCHER K, ROMANO R, SCHEURING J (2006) Wirtschaftsinformatik: Konzeption und Planung eines Informations- und Kommunikationssystems. Zürich: Compendio Bildungsmedien. $180 \mathrm{p}$.

LEMM R, ERNI V, THEES O (2006) Effiziente Kommunikation, Koordination und Kooperation im Netzwerk Holz dank IFIS - ein neues integriertes und webbasiertes Informationssystem. Schweiz Z Forstwes 157: 162-170. doi: 10.3188/szf.2006.0162

LEMM R, THEES O (2009) Unternehmensübergreifende Holzvermarktung mit der Koordinationsplattform IFIS UNO. In: Thees O,
Lemm R, editors. Management zukunftsfähige Waldnutzung. Grundlagen, Methoden und Instrumente. Zürich: VDF. pp. 499-520.

QUADE M (2007) Fallstudien Verein IFIS: Koordinationsplattform IFIS UNO. In: Wölfle R, Schubert $\mathrm{P}$, editors. Business Collaboration: Standortübergreifende Prozesse mit Business Software. Praxislösungen im Detail - Fallstudien - Konzepte - Modellierung. München: Carl Hanser. pp. 229-243.

SCHNETZLER M, LEMM R, BONFILS P, THEES O (2009) Das Supply Chain Operations Reference (SCOR)-Modell zur Beschreibung der Wertschöpfungskette Holz. Allg Forst- Jagdztg 180: 1-14.

STEPHAN MA, LEMM R, ZEHNDER D (2009) Der Verein IFIS. Ein innovatives Geschäftsmodell zur Vernetzung forstlicher Interessen im Bereich der Informationstechnologie. Thees $\mathrm{O}$, Lemm R, editors. Management zukunftsfähige Waldnutzung. Grundlagen, Methoden und Instrumente. Zürich: VDF. pp. 545-561.

Logiciel de logistique IFIS UNO: qu'avons-nous appris?

Le logiciel de logistique IFIS UNO soutient les processus commerciaux le long de la filière forêt-bois. En particulier, grâce à ce système, l'agrégation fastidieuse de l'offre en bois de la propriété forestière morcelée peut être optimisée et l'allocation des ressources en bois sur le marché mieux pilotée. IFIS UNO est maintenant utilisée depuis dix ans par différentes organisations de commercialisation de bois en Suisse. Ce système sera abandonné fin 2016, car d'importantes mises à jour qui ne peuvent pas être financées seraient nécessaires. Malgré cela, I'IFIS UNO peut être considéré comme une histoire à succès. C'est en partie grâce à ce système que les organisations de commercialisation de bois suisses ont pu s'établir comme réseaux de regroupement du bois rond centraux et reconnus. En parallèle, il a suscité des développements dans ce domaine très spécifique de logiciels. Nos expériences dans le développement et la mise en œuvre sont décrites dans cet article. D'une part, nous démontrons dans quels cas le développement d'un logiciel individuel est indiqué. D'autre part que l'importance de la phase de test dans le développement d'un logiciel individuel ne doit pas être sous-estimée, que la participation de l'utilisateur est indispensable et qu'il faut de la formation, du support et des mises à jour. Ces expériences, et bien d'autres encore, devraient être utiles aux intéressés développant des projets similaires. 